\title{
Integrating information security in the development of telematics systems
}

\author{
O. Tettero, D.J. Out, H.M. Franken, J. Schot \\ Telematics Research Centre (TRC) \\ P.O.Box 589, 7500 AN Enschede, The Netherlands \\ +31 53 4850485; \{Tettero, Out, Franken, Schot\}@trc.nl
}

\begin{abstract}
As organisations become aware of their vulnerability to threats to their information and telecommunication systems, this often results in the ad-hoc addition of safeguards to those systems. This causes operational problems, because information security requirements were never an issue during the development of these systems. In this paper we propose that requirements for information security should be integrated in the development process in an early phase. The benefit of the integration is that information security will become an integral part of the system. We discuss the complications and some preliminary guidelines, assuming that system developers are used to a 'traditional' development process.
\end{abstract}

\section{Keywords}

Telematics systems, system development, design, requirements, information security

\section{INTRODUCTION}

Organisations using telematics systems ${ }^{1}$ were generally unaware of their vulnerability to threats for a long time. For some the many incidents, public debates and governmental regulation have changed this. Unfortunately, information security requirements are difficult to effectuate in existing telematics systems. An example as illustration: ${ }^{2}$

\footnotetext{
${ }^{1}$ Telematics is the support of interaction between people and/or processes while bridging distance and/or time, through the integrated application of information and telecommunication technology.

2 The example results from a study identifying the conditions for large-scale application of telematics systems in the Netherlands, sponsored by the Ministry of Economic Affairs, (Breed et al., 1995).
} 
A Dutch University used an open network to exchange employee information between its central department and its annexes. To secure the information, crypto box-based encryption is used. The crypto-boxes are placed at strategic points in the network, to divide it in several secure and unsecure parts. The result was that the crypto-boxes caused a substantial loss of performance, both in the secure and especially in the unsecure part of the central hosts and servers. The key management system produced a large additional load for a network already plagued by heavy traffic.

In a number of similar cases, information security was added to existing telematics systems, which caused the following problems:

- Modification of an operational system may affect its functionality, resulting in a deviation from the desired functionality as defined in the user requirements of that system (Breed et al., 1995). The extent of the deviation is often unclear.

- Adding security to a system often causes changes in its use: organisational procedures surrounding that system should be changed as well, possibly causing all kind of changes within the organisation.

- The addition also results in huge costs (Mostert and von Solms, 1994). Often, the first addition of information security is unsatisfactory, and further additions must be made to deliver a secure system with identical functionality.

Tompkins and Rice (1986) and Booysen and Eloff (1995) foresee that taking information security into account from the start of the development process avoids problems later. However, we could not find a systematic description of a development process of telematics systems in the literature that actually has information security as one of its concerns.

\section{Contributions of this paper}

In this paper we investigate aspects of information security related to all stages of a traditional system development process. This process has a conceptual view of a system as input and the realisation of that system in an operational environment as output. We distinguish two viewpoints on the development activities: the method of development and the problem solving during the development. The method is a systematic procedure to obtain a real system (Dasgupta, 1989). This paper addresses the problem solving elements, like expressing the requirements. We focus on the development of safeguards as one of the concerns within the development process. Baskerville (1993) gives an analytical description of problem solving elements in the evolution of information systems. The authors are currently elaborating guidelines for several stages of the development process.

\section{Definitions}

A telematics system integrates information and telecommunication technology and consists of application software, system software, hardware and organisational procedures, Telematics systems contain assets, e.g. information, operate on assets or can be assets themselves. The system is operational in some environment. This environment contains a number of threats to the system. The assets are protected from threats by safeguards. Vulnerabilities in the system are weaknesses in the system or the 
absence of safeguards, whereas attacks are realisations of threats. Attacks that make use of the vulnerabilities are risks for the organisation and its systems (Ford, 1994).

Systems can be secured by applying information security ${ }^{3}$, which is a complex of measures and procedures that meets the requirements of confidentiality, integrity, availability and authenticity ${ }^{4}$ of systems in an adequate way, based on (Aalders et al., 1985), (BSI, 1994) and (OECD, 1996).

\section{SYSTEM DEVELOPMENT ELEMENTS}

To explore the incorporation of security in a development process, we need to understand its typical elements: the phases, the actor roles and conditions.

\section{Phases}

Several approaches to the development process are described in the literature, of which we used the common essentials, see e.g. (Mazza et al, 1994) and (Sommerville 1996). We have divided the process into phases ${ }^{5}$, as defined in Figure 1. This approach is commonly recognised as a top-down, step-wise refinement approach. It is used because it facilitates reasoning at different levels of abstraction. There may be several loops within a phase and in the development process. Loops in the development process result from decisions made in a certain phase, that turn out to be unfeasible in a later phase: in that case the development process has to back up to the earlier phase and redo the unfeasible decisions.

\section{Actors}

Actors in the field of security are bounded by the prescriptions, prohibitions and constraints of government and branch organisations. The constraints of the government affect the products sold on the telematics market, both by regulation of distribution of certain products, or prescribing minimum levels of security. The various actors, actor roles and their influence on security are shown in Figure 2.

We identify two classes of actor roles, whose types of behaviour influence the development process, i.e. the Telematics Market and the Organisation.

- The Telematics Market consist of the actor roles System Architect and System Builder. The first is involved in the creation of the design of the System. The second is involved when the SYSTEM is built and made operational.

- The Organisation is the purchaser of the SYSTEM and imposes requirements on the function and functioning of the SYSTEM. It can be a sole, autonomous institute, a part of a larger company or a consortium of several autonomous organisations. The Organisation consists of two sub classes, Management and Users.

- The Organisation Management directs and controls the course of the Organisation. The functioning of the Management is established in a policy, of which the security

\footnotetext{
${ }^{3}$ For short we use the term security for information security in the remainder of the paper.

${ }^{4}$ As telematics systems are heavily oriented towards communication, we introduce authenticity as a separate requirement, defined as traceability of origin and ownership of data in a system or a process.

${ }^{5}$ In this paper we refer to the system that is designed in the design process as SYSTEM.
} 


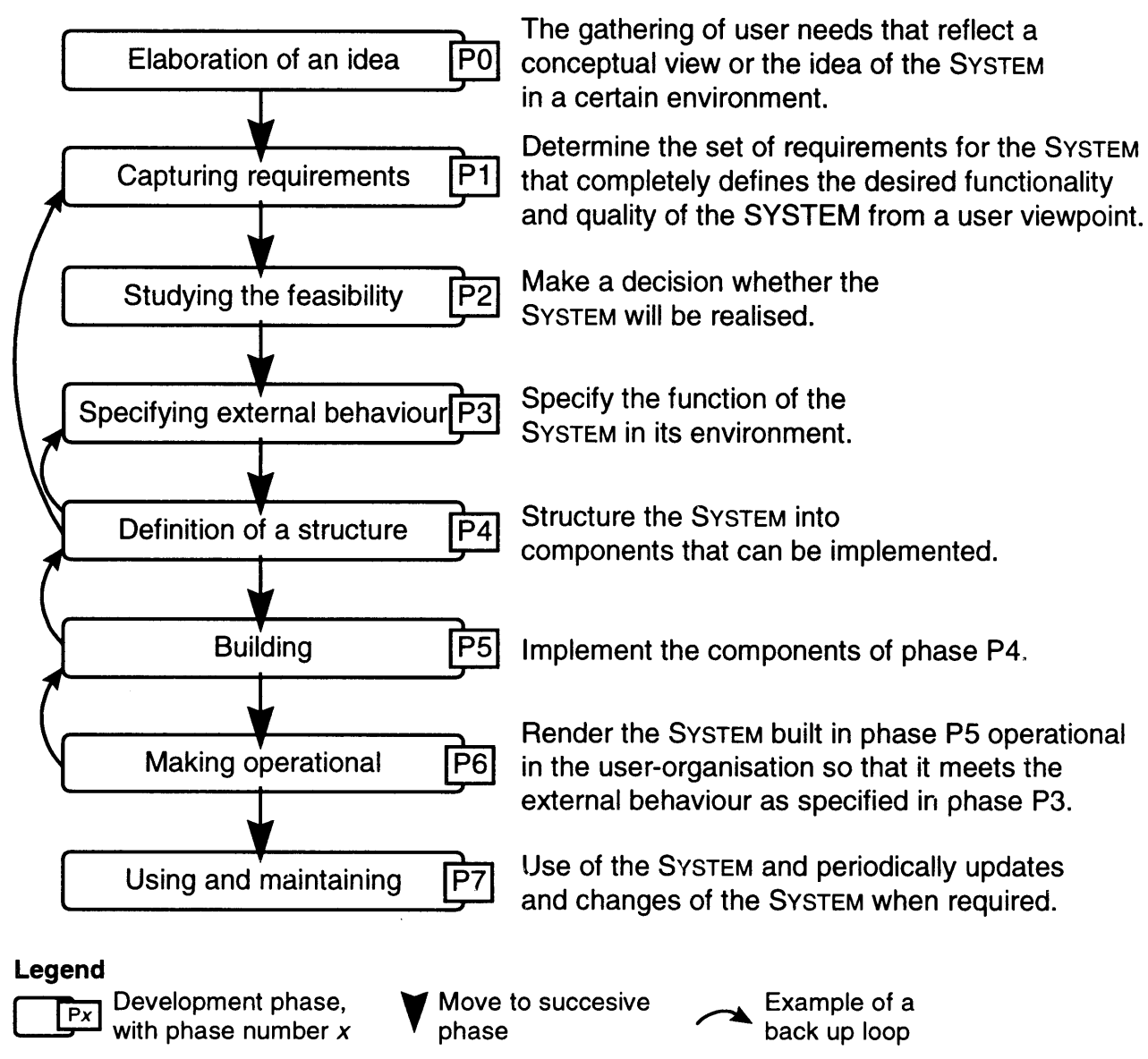

Figure 1 Phases and objectives of the traditional development process.

policy is a subset. The security policy is a set of rules to apply to all securityrelevant activities (Ford, 1994). This policy therefore constrains the development. We identify two actor roles within the sub class Management of the Organisation, i.e. the Top level Manager and the Security Officer. The first is responsible for all matters related to information technology and telecommunication, and is part of the upper management. The latter is responsible for security matters.

- We identify two actor roles within the Users of the Organisation, the System Administrator, responsible for control, administration and maintenance of the SYSTEM, and the End User, who makes hands-on use of the SYSTEM.

\section{Conditions}

When developing secure telematics systems, it is insufficient to consider the System separate from the Organisation. To develop a secure Sysтem for an Organisation, an actor in the Telematics Market should fulfil at least the following conditions, see e.g. (Parker 1981), (Fisher, 1984), (Badenhorst and Eloff, 1989) and (Hitchings, 1995):

- Establishment of a security policy by the Management of the Organisation;

- Identification of the assets of the Organisation that need to be protected;

- Identification of the risks for the Organisation; 


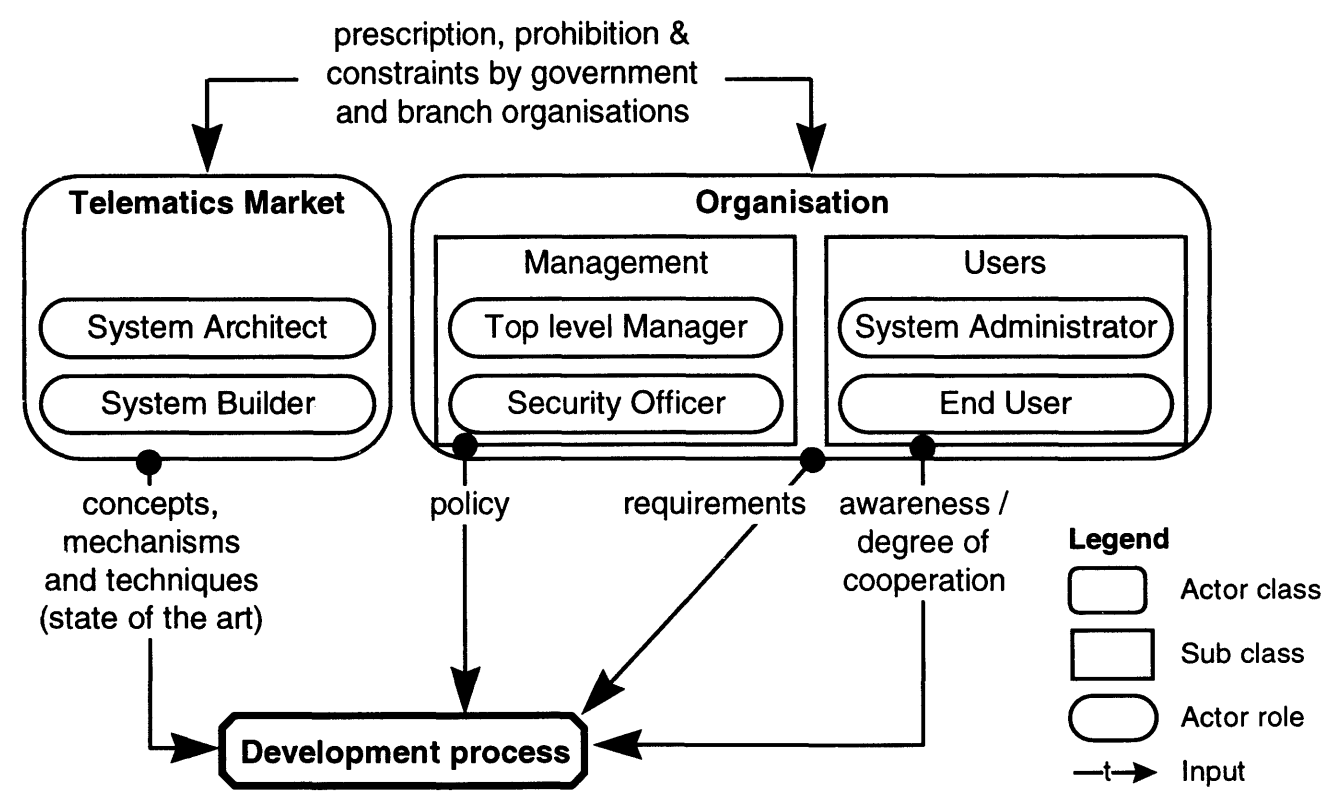

Figure 2 View on information security and a development process.

- Awareness of Management and Users of the need for security;

- Determination of which safeguards should be implemented where;

- Security is operational in an environment and therefore possesses not only technical dimensions, but economic, legal, organisational and human dimensions as well.

\section{SECURITY AND THE DEVELOPMENT PROCESS}

In this section we give an overview of the goals and characteristics of the phases in a traditional development process and we discuss the complications and preliminary guidelines of integrating security into this process (Tettero, 1996).

\section{Elaboration of an idea}

The goal of this phase is the gathering of user needs that reflect an idea of the SYSTEM capabilities in a certain environment. This idea can arise from many different situations, such as automating the communication process with another organisation, or the outcome of a business redesign process. The user needs are not yet clearly defined and precise. They express the purpose, scope and characteristics of the SYSTEM, and are laid down in a project proposal.

The idea of the SYSTEM can include security. However, this will not often be the case, because the core business of organisations is usually unrelated to security. In this paper we assume that the main purpose of a SYSTEM (we refer to this as to the "mustfunctionality') is not related to security, and therefore not part of the original idea. An example of an idea is a ticket vending machine that issues tickets for train passengers.

The user needs are a first elaboration of the idea and should contain suggestions for security issues. The suggestions should at least indicate that Management is aware of 
the importance of security for this System. If security is not included in the project proposal it will be difficult to assign resources (e.g. personnel) to investigate security issues.

\section{Capturing requirements}

The goal of this phase is to determine the set of requirements for the SYSTEM that completely defines the desired SYSTEM functionality and quality from a user-oriented viewpoint. The requirements defines what the SYSTEM should do and not how.

In the example of the ticket vending machine the following requirements could be captured:

- Single and return tickets;

- Every railway station in the country can be entered;

- Only the destinations can be given, station of departure is station of vending machine location;

- Payments by means of coins or an electronic system.

Security requirements are functional or indicate the quality level of these functional requirements. Definition of these requirements involves the following three issues:

1. Government and branch organisations demand that certain requirements concerning, a.o., privacy, computer criminality and encryption, are incorporated in the SYSTEM.

2. Security requirements are derived from the Organisation's security policy.

3. Users connect security requirements and resulting safeguards with unlikely events and expected unpleasant work procedures.

The gathering of security requirements is complicated, because they are often implicit (Mostert and von Solms, 1994). Security awareness, although increasing, is still not at an adequate level. The Users are focused on the SYsTEM must-functionality. Mostert and von Solms (1994) proposed a method to gather implicit requirements in which requirement engineers have knowledge of both the security and the SYSTEM domain. However, the security requirements can only be defined if the definition of the mustfunctionality is stable, otherwise nothing can be secured.

In the ticket vending machine example the implicit requirements are, amongst others:

- Tickets cannot be issued without proper payment;

- Only the ticket that a passenger has asked for should be issued;

- Money should not be removable from the machine.

The security requirements are based on threats. A threat analysis can support this, see e.g. (BSI, 1994), by comparing the costs of safeguards for these threats with the costs of an attack it is possible to determine which threats should be taken into account. CCTA (1991) developed risk analysis and management methodology (CRAMM) which analysis the threats and asset groups and determines countermeasures.

The operational SYSTEM environment strongly affects the security requirements. In a military environment, confidentiality requirements prevail over other security requirements, while in a business environment the integrity requirements prevail (Clark and Wilson, 1987). Other environmental influences are e.g. use of public networks and the controllability of Users by the Organisation. 


\section{Studying the feasibility}

The goal of this phase is decide whether the SYSTEM will be realised. The advantages, disadvantages, costs and benefits of the requirements are considered against the financial, organisational and technical possibilities of the Organisation and the Telematics Market.

Security is part of the requirements that are subject of the final negotiations between Organisation and System Architect. These requirements require insight in the possible safeguards and in their usage or nonusage once the SYSTEM is operational.

\section{Specifying external behaviour}

The goal of this phase is to (formally) specify the function of the SYSTEM in its environment. In contrast with phase P1 the function is specified from the SYSTEM viewpoint. The external behaviour only specifies what the SYSTEM should do within its environment.

The specification consists of the SYSTEM function, the (user) interfaces or interactions with the environment and the Quality of Service-measures (e.g. response times, throughput) delivered by the function. Contents of the specification are e.g. activities and services offered to the environment, SүSTEM boundaries and representation of data.

In the example of the ticket vending machine the following behaviour is specified:

- Clear all choices; $\quad$ Provide means for the input of the type of ticket:

- Issue the ticket;

- Provide means for the payment of the ticket;

- Provide means for the input of the destination;

- Show all possible destinations and possible tickets; - Show the price of the selected ticket;

By specifying, the SYSTEM boundaries are defined by means of the interfaces with the environment and the knowingly allowed interactions. The term interface should be regarded in the broadest sense of the word. For instance, damaging the ticket vending machine with a hammer. The interface here is the outside plate of the ticket vending machine. The SYSTEM boundaries are defined as appropriate for this stage, however for a different development phase a different boundary can be appropriate. An example is the power supply for the ticket vending machine. In the current phase it could be regarded as an internal aspect of the SYSTEM, while in the building phase an external source is used. The introduced threat is: the power supply can simply be interrupted by pulling o't the plug.

The main complication for security is that the SYSTEM is formalised and predictable whereas the SYSTEM environment is unpredictable. A solution is to view threats as actions in the environment, and attacks can then be viewed as interactions with the SYSTEM.

The traditional development process focuses on realising the must-behaviour of SYSTEMS. For security we also address misbehaviour of the SYSTEM or parts thereof, and exceptional behaviour of the SYSTEM environment. Normally, interfaces are precisely defined. However, it is impossible to define an interface when behaviour is not well known. The realised SYSTEM should be able to cope with behaviour that falls outside the prescriptive environment view in the specifications. 


\section{Definition of a structure}

The goal of this phase is to structure the SYSTEM into components that can be implemented. The resulting structure is a hierarchical set of components, each of which is labelled with a function and its interfaces. Every component of the lowest level can be implemented by a System Builder. The whole of components and interfaces performs the function as specified in phase P3.

The SYSTEM structure supports the System Architect to keep track of the consequences of changes in the design. Especially for security a good structure is needed, because changes in the design can imply changes for all components or interfaces. The design of a good structure is based on a set of principles and techniques, which guide the process of decomposition regarding security (Muftic, 1994).

Security can be defined in the structure in two ways:

- Independent component. This component is fully dedicated to security, for example an authentication service. Other components can neglect the security components or can use its functionality.

- Incorporated in all components. Every component has some functionality, for example confidentiality functionality.

The International Standardisation Organisation has proposed standard services for a high level structure based on the OSI reference model (ISO, 1989).

\section{Building}

The goal of this phase is to implement the components of phase P4. Hardware components should be bought or physically built, while software components should be bought or programmed. Individual hardware and software elements must be connected and tested as a group. The SYSTEM has to be tested and documented.

To realise the security part of the components, safeguards are available, of which only the technical part is implemented in this phase. For proper implementations of safeguards the following should be considered:

1. Impact on the organisation. The characteristics of a safeguard determine the type of procedures in the Organisation that should be used. The impact can be the workload for End Users, changes in the organisational structure or extra costs.

2. Legitimacy of the technical safeguards. Some countries have stringent export controls. Products falling under these controls and produced in that country cannot be exported without government permission. Certain technical safeguards fall under these regulations and thus cannot always be freely used.

The System Builder can buy standard safeguards on the Telematics Market to realise the SYSTEM, such as a plug-in crypto card. Using standard elements can reduce costs of the SYSTEM. The standards elements have a certain function and interface and are meant to operate in a certain type of environment. The standard safeguard will probably not fully comply in function, quality and interface of the desired safeguard for the SYSTEM. To make the standard safeguard useful in a wide range of systems, its interface will be general and will thus include interactions that the SYSTEM does not need or that may introduce potential weaknesses. 


\section{Making operational}

The goal of this phase is to render the SysTEM built in phase P5 operational in the Organisation so that it meets the external behaviour as specified in phase P3. The SYSTEM from phase P5 is built and tested under 'laboratory conditions' and must be tuned to the Organisation and integrated into existing systems. In addition, new procedures in the Organisation should be set up, or existing procedures should be modified.

The technical safeguards of the SYSTEM are extended with organisational procedures. Procedures are needed because organisations change rapidly, while the technical SYSTEM is fixed. The SYSTEM must be used in the daily work of an End User, for which the daily work procedures should be adjusted.

The SYSTEM introduced to the Organisation should comply with the Organisation policies, including the security policy. However, the SYSTEM introduces new qualities which means that the security policy possibly has to be adjusted.

\section{Using and maintaining}

Once the System is made operational in phase P6, it should be ready to be used for the goals of that moment in the organisation of that moment. Since both the goals and the organisation are susceptible to change, and no system is without bugs, the SYSTEM will have to be updated and changed periodically. Changes that affect the SYSTEM can be the cause of:

1. Predictable facts of life, e.g., the absence of people and career moves;

2. Organisation policies changes, e.g. introduction of EDI;

3. Environment changes, e.g. new laws or technology / policy changes of competitors.

Changes resulting from predictable facts of life should be anticipated in adequate organisational procedures. In this phase, the procedures and related data are carried out and kept up-to-date. An example is the provision of a password to a new employee, its removal when he leaves, its periodical update, and related events. Changes in the policies of an organisation could demand changes in the SYSTEM requirements and therefore cause adjustments. When the changes involve security these should be treated just as the other requirements. Changes in the environment should be studied carefully, since these could introduce new threats, requiring new safeguards in the SYSTEM. Changes could adjust the requirements and introduce other relations to the security requirements.

By using the System, the End Users get to know the SYSTEM well and will discover deficiencies. End Users will become aware of security bugs. These bugs cannot be repaired immediately. The (multiple) causes of bugs can be found in indistinct procedures, malicious techniques and design flaws. Their consequences should be investigated carefully. To remove the bugs from the SYSTEM, the SYsTEM should be updated.

\section{Guiding the whole development process}

If the requirements include security, it is yet unclear how these additional requirements proliferate throughout the remaining development phases. For example, existing security evaluation criteria, such as ITSEC (Europe) and TCSEC (USA), could be 
incorporated as requirements for the development of a secure system. This means that the realised SYSTEM should pass a particular security level check as defined in these public criteria. The question is how these requirements translate into design and implementation decisions.

Landwehr et al. (1994) and Muftic (1994) recognise that maliciously introduced faults are not to be expected in the requirements, because they have been thoroughly reviewed. However, it is still possible to compromise the requirements in the remainder of the development process.

Specifications can be compromised unintentionally or intentionally. Unintentionally compromises follow from decisions made when filling in the degrees of freedom of previous phases or from too little insight in the consequences of the decisions. Examples of this kind of decisions are speed, simplicity and implement shortcuts. Intentionally compromises can be introduced by System Builders by putting backdoors in the System, like Trojan horses (Landwehr et al., 1994). These compromises are far more difficult to avoid.

To escape from compromising the specifications, security should be maintained throughout all phases of the development process (Mostert and von Solms, 1994). Therefore, every phase should have additional, identifiable security conditions.

The tension between abstraction and completeness in different stages of the development can cause problems (Boswell, 1995). Every developrnent phase represents the SYSTEM at a different level of abstraction. Sometimes knowledge of (technical) safeguards is needed in an early stage of the development process where this kind of knowledge normally is ignored. As a consequence a top-down development strategy alone will never be sufficient in practice.

\section{CONCLUSIONS}

Adding safeguards to operational telematics systems leads to operational problems and to systems that are not secure. To avoid these problems information security should be integrated in the development process of telematics systems. However, information security is not a common concern to take into account. Difficulties for the integration are the following:

- Traditional development focuses on the must-functionality of the SySTEM;

- Current requirements capturing methods only gather the explicit user needs. Since information security requirements are often implicit, these remain hidden;

- Information security requirements are the first to be withdrawn in case of conflicting requirements;

- Prescription of the behaviour of SYSTEM environment alone is insufficient, because this behaviour is unpredictable, unknown and contributory to the SYSTEM behaviour;

- Design decisions can compromise information security specifications;

- The use of technical safeguards is constrained by the impact on the organisation and their legitimacy;

- It is unclear how information security requirements proliferate throughout the development process regarding potential malicious behaviour and selection of safeguards regarding the tension between abstraction and detail. 
Considering the above difficulties we recommend the following:

- Decisions as to which requirements prevail over others should be guided by a strategy, based on the security policy of the Organisation;

- The SYSTEM environment should be taken into account in the definition of the development, because of its impact on the requirements;

- Development of methods or frameworks to translate a security policy to SYSTEM requirements, gather implicit requirements, include the behaviour of the environment (e.g. threats) in the specifications of interactions between the SYSTEM and its environment, reflect information security dimensions in the SYSTEM structure and define appropriate SYSTEM boundaries at every level of abstraction;

- Development of a set of information security principles to guide the structuring of a SYSTEM that helps to study the consequences of design decisions, to reduce complexity and supports comprehension of the interactions among parts of the SYSTEM;

- Definition of guidelines and techniques to map information security evaluation criteria onto practical guidelines for every development phase and to maintain information security throughout the whole development process.

\section{Acknowledgements}

This paper is partly based on research in the PLATINum/Mesh project iLucent Technologies, TRC \& CTIT), which is funded by the Dutch Ministry of Economic Affairs. PLATINUW/MESH aims at the design of a multimedia broadband ISDN platform.

\section{REFERENCES}

Aalders, J.C.H., Herschberg, I.S. and Zanten, A. van (1985) Handbook for infformation security: a guide towards information security standards. Elsevier Science.

Badenhorst, K.P. and Eloff, J.H.P. (1989) Framework of a methodology for the lifecycle of computer security in an organisation, in Computers \& Security, 8, 5.

Baskerville, R. (1993) Information systems security design methods: implications for information systems development, in Computing Surveys 24 (4).

Booysen, H.A.S. and Eloff, J.H.P (1995) A methodology for the development of secure application systems, in Information security - the next decade : Proceedings of IFIP Information security. (ed. J.H.P. Eloff, von Solms S.H.), Chapman \& Hall, London.

Boswell, A. (1995) Specifications and validation of a security policy model. IEEE Transactions on Software Engineering, 21, 2.

Breed, N.F., Out, D.J. and Tettero, O. (1995) Informatiebeveiliging, een blik achter de schermen. Samsom BedrijfsInformatie, Alphen a/d Rijn/Zaventem. [In Dutch]

BSI - British Standard Institute (1994) Code of practice for information security management.

CCTA (1991) SSADM-CRAMM Subjectguide for SSADM version 3 and CRAMM version 2. Central Computer and Telecommunications Agency, IT Security and Privacy group, Her Majesty's Government, London.

Clark, D.D. and Wilson, D.R. (1987) A comparison of commercial and military computer security policies, in Proceedings of Symposium on Security and Privacy. 
Dasgupta, S. (1989) The structure of design processes, in The structure of design processes. (ed. M.C. Yovtis), volume 28 of Advances in computers.

Fisher, R.P. (1984) Information systems security. Prentice Hall, Engelwood Cliffs.

Ford, W. (1994) Computer communications security: principals, standard protocols and techniques. Prentice Hall, New Jersey.

Hitchings, J. (1995) Deficiencies of the traditional approach to information security and the requirements for a new methodology, in Computers \& Security, 14.

ISO/TC 97 (1989) Information processing systems - Open Systems Interconnection Basic Reference Model - Security Architecture. ISO 7498-2.

Landwehr, C.E., Bull, A.R., McDermott, J.P. and Choi, W.P. (1994) A Taxonomy of computer program security flaws, in ACM computing surveys, 26, 3.

Mazza, C., Fairclough, J., Melton, B., Pablo, D. de, Scheffer, A.. and Stevens, E. (1994) Software engineering standards. Prentice Häl/European Space Agency.

Mostert, D.N.J. and von Solms, S.H., (1994) A methodology to include computer security, safety and resilience requirements as part of the user requirement, in Computers \& Security, 13, 4.

Muftic, S. (1994) Security architecture for ODP systems, final results of the CEC COST-11 Ter "Security" project, in Computer Networks and ISDN Systems, 26.

OECD - Organisation for Economic Co-operation and Development (1996) Guidelines for the security of information systems.

Parker, D.B. (1981) Managers guide to computer security. Reston Publishing.

Sommerville, I. (1996) Software engineering. Addison-Wesley, 5th ed.

Tettero, O. (ed.) (1996) Security aspects of telematics applications, PlatiNUM deliverable D3.2. Telematics Research Centre, Enschede.

Tompkins, F.G. and Rice, R. (1986) Integrating security activities into the software development lifecycle and the software Quality assurance process, in Computers \& Security, 5.

\section{BIOGRAPHIES}

Olaf Tettero is associate member of scientific staff of the TRC. He holds an M.Sc. in computer science. His current research interest focuses on information security and design processes. He works on the design of a secure multimedia broadband platform.

Dirk Jan Out holds an M.Sc. and Ph.D. in computer science. He works as a member of the scientific staff of the TRC. His current research interest focuses on teleeducation, information security and the design of multimedia broadband platform.

Henry M. Franken holds an M.Sc. and Ph.D. in Electrical Engineering. He works as a member of the scientific staff of the TRC. His current research interest focuses on applying systems engineering principles to telematics and business process (re)design.

Jeroen Schot is senior staff member of the TRC. He holds a BSc, MSc and PhD in computer science. His research interests are distributed systems design, and methods for supporting this. He has been involved in many projects in the health care, mobile telecommunications, space and aviation, logistics, and software development sectors. 